\title{
The influence of the cholesterol microenvironment in tissue sections on molecular ionization efficiencies and distributions in ToF-SIMS
}

\author{
A.F. Maarten Altelaar ${ }^{\mathrm{a}}$, Jan van Minnen ${ }^{\mathrm{b}}$, \\ Ron M.A. Heeren ${ }^{\text {a }}$, Sander R. Piersma ${ }^{a}, *$ \\ ${ }^{a}$ FOM Institute for Atomic and Molecular Physics, Kruislaan 407, 1098 SJ Amsterdam, The Netherlands \\ ${ }^{\mathrm{b}}$ Department of Molecular and Cellular Neurobiology, Faculty of Earth and Life Sciences, Vrije Universiteit, \\ De Boelelaan 1085, 1081 HV Amsterdam, The Netherlands \\ Received 12 September 2005; accepted 15 February 2006 \\ Available online 2 May 2006
}

\begin{abstract}
High-resolution images of cholesterol were obtained from Lymnaea stagnalis nervous tissue using metal-assisted (MetA) time-of-flight secondary ion mass spectrometry (ToF-SIMS). The spatial distributions of different pseudomolecular ions of cholesterol $[M-\mathrm{H}]^{+},[M-\mathrm{OH}]^{+}$ and $[2 M+\mathrm{Au}]^{+}$, illustrate the influence of the tissue microenvironment on the ionization efficiencies of these ions. These biological matrix effects result in differences in localizations of molecular ions derived from the same molecular species.
\end{abstract}

(C) 2006 Elsevier B.V. All rights reserved.

Keywords: Metal-assisted; ToF-SIMS; Imaging; Cholesterol; Nervous tissue

\section{Introduction}

Cholesterol is one of the major constituents of the plasma membrane $(\sim 30-40 \mathrm{~mol} \%)$, where it plays a crucial role in membrane dynamics, organization and function [1-3]. In recent years, it has become apparent that elevated levels of cholesterol are associated with several diseases like Alzheimer's disease and atherosclerosis [4,5]. In Alzheimer's disease it has been shown that altered cholesterol distributions within neurons play an important role $[5,6]$. Although the brain is the organ with the highest amount of cholesterol ( $\sim 20 \%$ of total body cholesterol), the analysis of cholesterol distributions has been technically challenging [5].

Imaging secondary ion mass spectrometry (SIMS) provides both chemical specificity and spatial distributions of components present at a surface. SIMS can routinely achieve sub-micron spatial resolution [7], using traditional primary ion beams like $\mathrm{Ga}^{+}$, but the sensitivity for high-mass ions is limited [8]. In order to enhance the ionization yield of large intact molecular ions in SIMS, different kinds of sample surface coating (MALDI

\footnotetext{
* Corresponding author. Tel.: +31 20 6081394; fax: +31 206684106 . E-mail address: piersma@amolf.nl (S.R. Piersma).
}

matrices [9-13], silver [14,15] and gold [16-20]) as well as the use of polyatomic primary ion beams [21-26] have been reported. We recently showed the usefulness of matrix-enhanced (ME) SIMS in direct molecular imaging of biological tissue at subcellular resolution [12,13] as well as in the analysis of phospholipids [11] and the use of sample metallization in the SIMS analysis of single neuroblastoma cells [27].

Several recent studies point to preferential ionization of lipid and sterol species in SIMS imaging of biological surfaces $[12,14,15,24,25,27]$. Imprinting tissue on silver coated surfaces, metallization of biological surfaces with silver or gold and the use of polyatomic primary ion beams all result in protonation or cationization of surface lipids and sterols. These studies show that SIMS imaging might play an important role in answering questions concerning the distribution of these species in biological surfaces. Even more so since the conventional approach to study the spatial behavior of these molecules (fluorescence microscopy) has been hampered by technical challenges [5,28]. Having said this, one has to be careful with the interpretation of the obtained SIMS images from biological surfaces. Sostarecz et al. [29] have already shown that the environment where the molecule of interest is present is of great influence on ionization yields. The addition of a proton donating species (cholesterol) to a Langmuir-Blodgett thin film of 
dipalmitoyl phosphatidylcholine greatly altered the lipid ionization efficiency. One can imagine that with the complexity of real biological samples like tissue sections this phenomenon will be even more important. In this study, we show high spatial resolution molecular distributions of cholesterol in nervous tissue obtained with metal-assisted (MetA) SIMS. We show that both the absolute and relative ionization efficiencies for the cholesterol ions $[M-\mathrm{H}]^{+},[M-\mathrm{OH}]^{+}$and $[2 M+\mathrm{Au}]^{+}$in tissue sections is a function of spatial location.

\section{Experimental}

\subsection{Materials}

Cholesterol and acetone were purchased from SigmaAldrich (Zwijndrecht, The Netherlands).

\subsection{Tissue sampling protocol}

Freshwater snails (Lymnaea stagnalis) were raised under laboratory conditions; $20 \pm 1{ }^{\circ} \mathrm{C}$ water temperature, 12-h light:12-h dark cycle and fed lettuce ad libitum [30]. Adult specimens were decapitated and the cerebral ganglia and connecting commissure were dissected, directly embedded in $10 \%$ gelatine (De Twee Torens, Delft, The Netherlands) at $30{ }^{\circ} \mathrm{C}$ and frozen at $-80{ }^{\circ} \mathrm{C}$. Gelatine embedding allows cryostat sectioning down to $5 \mu \mathrm{m}$ thickness and does not interfere with SIMS analysis. Ten micrometer tissue sections were cut on a Leica CM 3000 cryostat (Leica Microsystems, Nussloch, Germany) at $-20{ }^{\circ} \mathrm{C}$. Sections were picked-up on IndiumTinOxide (ITO, 4-8 $\Omega$ resistance) coated glass slides (Delta Technologies, Stillwater, MN), dried in a stream of warm air for $1 \mathrm{~min}$ and then quickly frozen on dry ice and stored at $-80{ }^{\circ} \mathrm{C}$. Conservation of morphology was checked by optical microscopy during the sectioning, drying and storage process. No obvious ice crystal damage was observed after freezing at $-80{ }^{\circ} \mathrm{C}$ in gelatine. Light microscopy images were acquired using a Leica DMRX microscope with a Nikon DXM1200 digital camera. Prior to mass spectrometry, frozen tissue sections were brought to room temperature in a desiccator over a silica gel canister.

\subsection{Gold deposition}

Gold was sputter coated on the sample surface using a Quorum Technologies (Newhaven, East Sussex, U.K.) SC7640 sputter coater equipped with a FT7607 quartz crystal microbalance stage and a FT7690 film thickness monitor.

\subsection{Mass spectrometry}

All static SIMS experiments were performed on a Physical Electronics (Eden Prairie, MN) TRIFT-II time-of-flight SIMS (ToF-SIMS) equipped with an ${ }^{115} \mathrm{In}^{+}$liquid metal ion gun, as described before [31]. Secondary ions were extracted through a $3.2 \mathrm{keV}$ electric field into the ToF analyzer and post-accelerated by an additional $8 \mathrm{keV}$ prior to detection on a dual multi- channel plate/phosphor screen detector. In the SIMS experiments, charge compensation was achieved by flooding the sample with low energy electrons $(29 \mathrm{eV}, 40 \mathrm{nA}, 2 \mathrm{~mm}$ diameter spot) between each primary ion pulse. To obtain images the focused ion beam was rastered across the sample. All experiments were performed with a primary ion beam current of $450 \mathrm{pA}$, a primary pulse length of $30 \mathrm{~ns}$, a spot diameter of $500 \mathrm{~nm}$ and a primary ion energy of $15 \mathrm{keV}$. The ion dose was such that all analyses were conducted in the static SIMS regime. The positive ion mode mass spectra and chemical images were taken from the same $150 \mu \mathrm{m} \times 150 \mu \mathrm{m}$ areas within the sample. In order to image a larger section, the sample stage was stepped in a mosaic pattern and the resulting images were aligned to produce a single molecular image.

\section{Results and discussion}

A MetA-SIMS spectrum of cholesterol spotted on a goldcoated $(5 \mathrm{~nm})$ silicon wafer, obtained with a $15 \mathrm{keV}{ }^{115} \mathrm{In}$ primary ion beam, is shown in Fig. 1A. The pseudo-molecular ion $[M-\mathrm{H}]^{+}$at $m / z 385$ and the dominant cholesterol fragment $[M-\mathrm{OH}]^{+}$at $m / z, 369$ can be clearly seen. In addition to these high intensity cholesterol peaks also several gold cationized species can be observed with the most dominant peak at $\mathrm{m} / \mathrm{z}$ 970, corresponding to the $[2 M+\mathrm{Au}]^{+}$species. In Fig. $1 \mathrm{C}$ and $\mathrm{D}$, the spectra of two imaging SIMS experiments of L. stagnalis nervous tissue are shown, measured with the same $15 \mathrm{keV}^{115} \mathrm{In}$ primary ion beam. In Fig. 1C, the SIMS experiment was conducted directly on the Lymnaea tissue without any surface modifications. The spectrum shows several low mass fragments, the phosphocholine headgroup fragment at $\mathrm{m} / \mathrm{z} .184$ and several higher mass species. All of the species in the higher mass range are either lipids between $\mathrm{m} / \mathrm{z}, 700$ and 800, lipidderived species (diacylglycerols and ceramides) between $\mathrm{m} / \mathrm{z}$ 500 and 700 or sterols, the cholesterol fragment at $m / z, 369$ and Vitamin E at $m / z$ 430. In Fig. 1D, a similar Lymnaea nervous tissue section is examined by SIMS after the deposition of $1 \mathrm{~nm}$ of gold on top of the surface, using a sputter coater. It can be seen that the spectrum drastically changes, with a strong enhancement of the cholesterol signals, the appearance of several gold cationized cholesterol peaks and a decrease (or even disappearance) of the peaks from the lipids and lipid derived species. The enhancement for the dominant cholesterol fragment at $m / z 369$, after gold deposition is two orders of magnitude while the enhancement for the pseudomolecular ion at $m / z 385$ is even slightly higher. Also, in the MetA SIMS tissue analysis the gold cationized species at $\mathrm{m} / \mathrm{z} 970$ is very dominant.

Fig. 1E-H shows the MetA-SIMS images of the Lymnaea nervous tissue sections after $1 \mathrm{~nm}$ gold deposition. The images were obtained from the commissure, connecting the two cerebral ganglia, cut in the axial plane using a cryomicrotome, as shown in Fig. 1B. Fig. 1E and F show the spatial distribution of two cholesterol derived peaks, namely the $[M-\mathrm{H}]^{+}$peak at $\mathrm{m} / \mathrm{z} 385$ and the fragment peak at $\mathrm{m} / \mathrm{z}$ 369. Although the two images depict the spatial distribution of the same molecule, they show remarkable differences, as can be seen in the overlay 

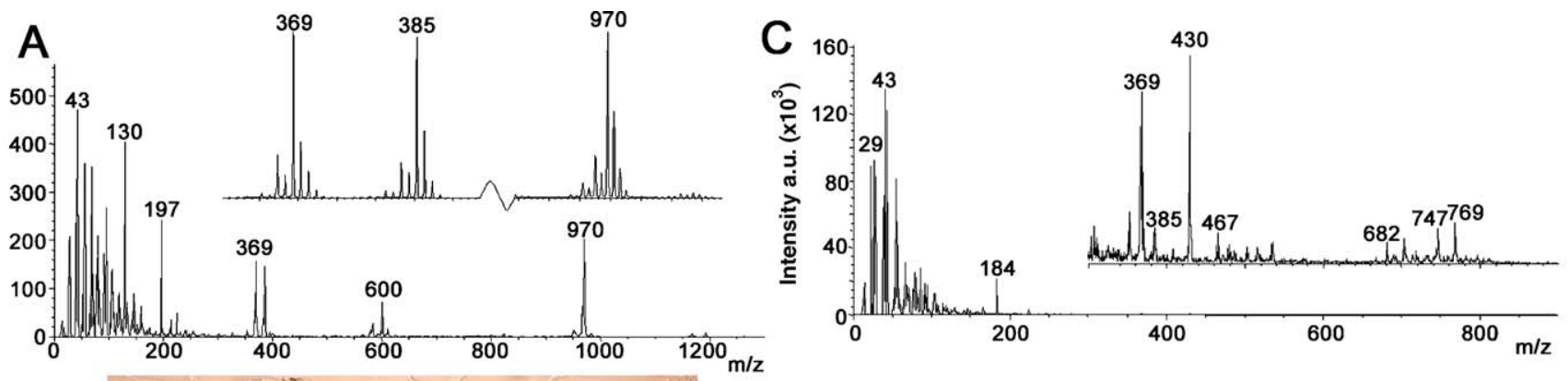

B

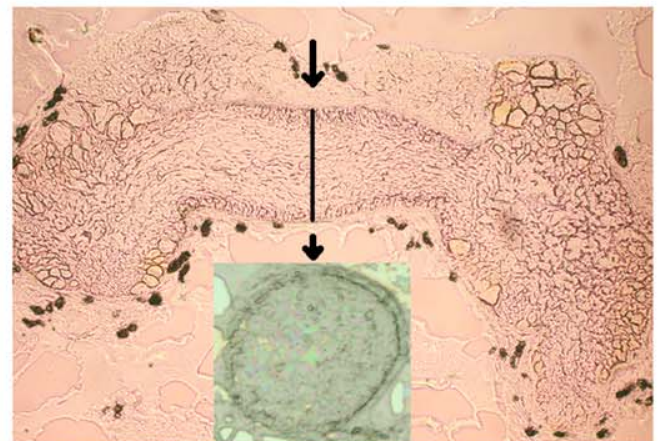

D

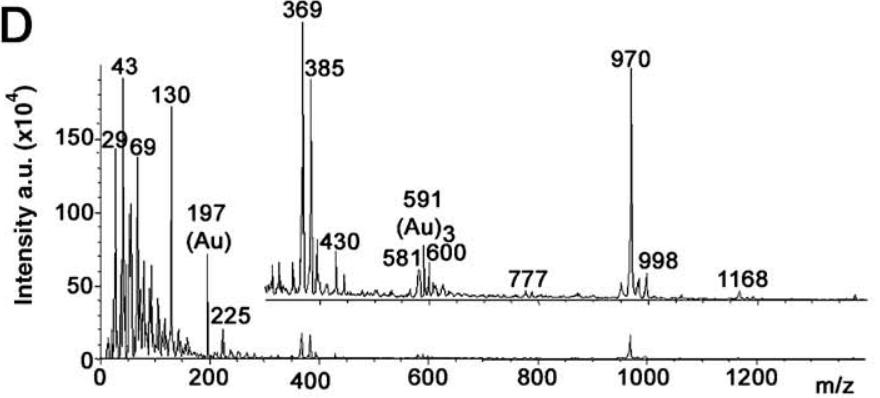

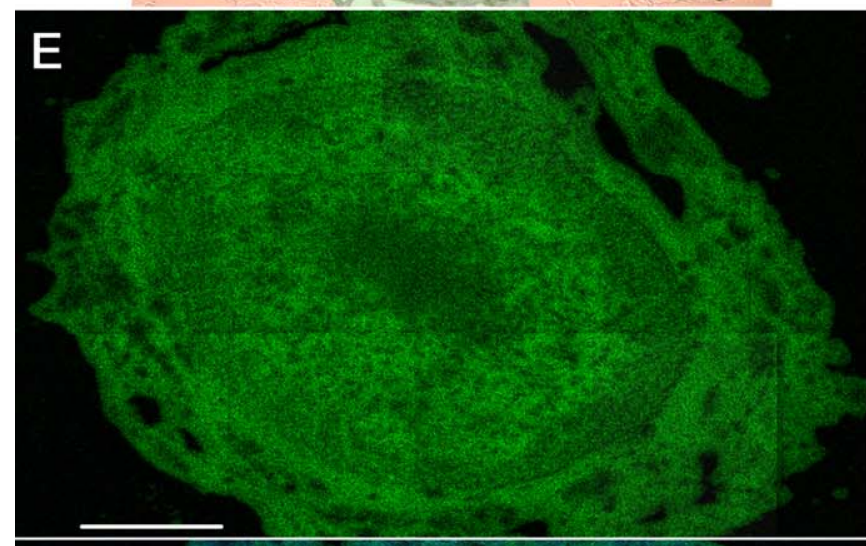
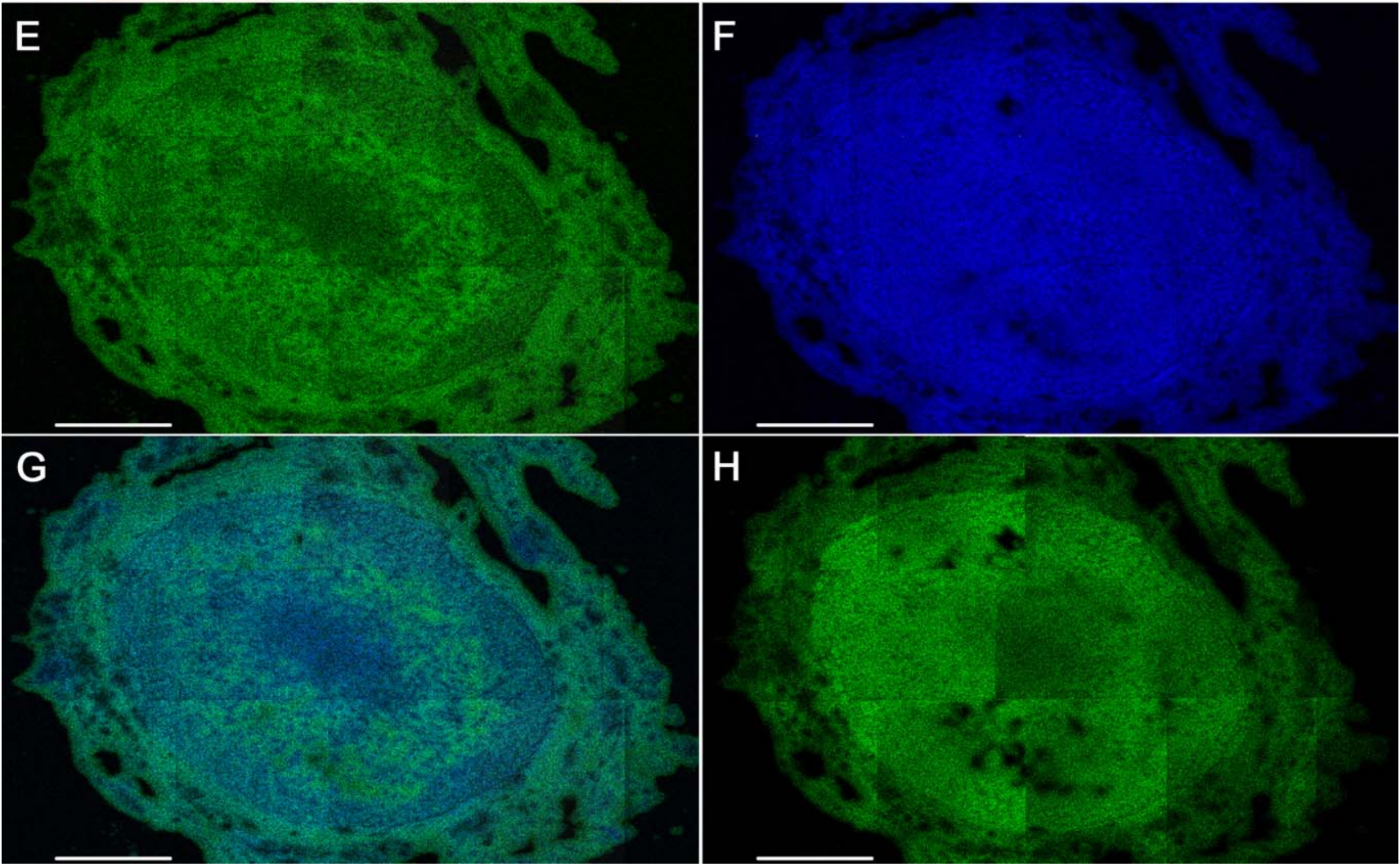

Fig. 1. SIMS imaging of cholesterol and Lymnaea stagnalis nervous tissue with (A) MetA-SIMS spectrum of cholesterol spotted on a gold coated (5 nm) silicon wafer (high mass range enhanced by factor of 2.5). (B) Optical microscopy image of Lymnaea stagnalis nervous tissue showing the left and right cerebral ganglia and the connecting commissure, inset shows cryomicrotome section of the commissure used in the MetA-SIMS analysis. (C) SIMS spectrum and (D) MetA-SIMS spectrum of Lymnaea stagnalis nervous tissue (high mass range enhanced by factor of 200 and 10, respectively). (E) MetA-SIMS image of cholesterol pseudomolecular ion $[M-\mathrm{H}]^{+}$distribution $\left(\mathrm{m} / \mathrm{z} 385\right.$, green, 0-20 counts). (F) MetA-SIMS image of cholesterol fragment ion $[M-\mathrm{OH}]^{+}$distribution $(\mathrm{m} / \mathrm{z}$ 369 , blue, 0-16 counts). (G) Overlay image of cholesterol pseudomolecular ion $[M-\mathrm{H}]^{+}\left(\mathrm{m} / z 385\right.$, green, $0-20$ counts) and cholesterol fragment ion $[M-\mathrm{OH}]^{+}$ distributions $\left(\mathrm{m} / \mathrm{z} 369\right.$, blue, 0-25 counts). (H) MetA-SIMS image of cholesterol pseudomolecular ion distribution $[2 \mathrm{M}+\mathrm{Au}]^{+}(\mathrm{m} / z$ 970, green, $0-5 \mathrm{counts})$. Scale bars are $100 \mu \mathrm{m}$. 
image of Fig. 1G. Looking at the image of the $m / z 369$ signal of cholesterol, the highest abundance can be found at the border of the oval structure and in the centre. Conversely, the $\mathrm{m} / \mathrm{z} 385$ signal of cholesterol shows the highest intensity as an oval ring between the border and the center of the commissure tissue section. It is known from both optical microscopy and electron microscopy studies that the commissure consists of two morphological compartments, separated by a sheath of glial cells [32]. The neurohaemal area of the caudodorsal cells forms the outer compartment, while the inner compartment consists of thousands of axons. Furthermore, ventral caudodorsal cells send axons through the inner compartment [32]. Inspecting the images in Fig. 1E and F, and the overlay in Fig. 1G, these inner and outer compartments as well as the separating sheath of glial cells can be clearly distinguished. The signal intensity of the $\mathrm{m} /$ $z 385$ signal is most intense at the glial cell area, separating the two compartments, while the $\mathrm{m} / z 369$ peak shows the highest intensity at the inner compartment and increased signal intensity at the outer compartment. It is clear from these results that the microenvironment surrounding the molecule of interest plays a crucial role in its ionization. Taking either one of the cholesterol-specific peaks to illustrate its distribution would give a false image. Furthermore, quantitative statements on cholesterol concentrations in these highly heterogeneous biological surfaces will be prone to errors, since matrix effects on the ionization efficiencies have to be taken into account.

In Fig. 1H, we show the spatial distribution of the $[2 M+\mathrm{Au}]^{+}$ion of cholesterol at $\mathrm{m} / \mathrm{z}$ 970. The image shows a different distribution of the pseudomolecular ion than in Fig. 1E and F. The image seems to be a convolution between the first two images, showing a decrease of signal intensity in the centre of the tissue and more or less homogeneous signal intensity through the rest of the tissue.

\section{Conclusions}

MetA-SIMS was used to obtain high-resolution images of different pseudomolecular ions of cholesterol. The results show that the abundances of the differently ionized species are influenced by the tissue microenvironment. The nature of the microenvironment composition, as well as its influence on ionization efficiencies needs to be determined for quantitative cholesterol imaging.

\section{Acknowledgments}

This work is part of research program no. 49 "Mass spectrometric imaging and structural analysis of biomacromolecules" of the "Stichting voor Fundamenteel Onderzoek der Materie (FOM)", which is financially supported by the
"Nederlandse organisatie voor Wetenschappelijk Onderzoek (NWO)".

\section{References}

[1] L. Liscum, N.J. Munn, Bba.-Mol. Cell Biol. L. 1438 (1999) 19-37.

[2] K. Simons, E. Ikonen, Science 290 (2000) 1721-1726.

[3] T.V. Kurzchalia, S. Ward, Nat. Cell Biol. 5 (2003) 684-688.

[4] A.J. Lusis, Nature 407 (2000) 233-241.

[5] L. Puglielli, R.E. Tanzi, D.M. Kovacs, Nat. Neurosci. 6 (2003) 345-351.

[6] L. Puglielli, G. Konopka, E. Pack-Chung, L.A.M. Ingano, O. Berezovska, B.T. Hyman, T.Y. Chang, R.E. Tanzi, D.M. Kovacs, Nat. Cell Biol. 3 (2001) 905-912.

[7] P.J. Todd, J.M. McMahon, R.T. Short, C.A. McCandlish, Anal. Chem. 69 (1997) A529-A535.

[8] I.S. Gilmore, M.P. Seah, Int. J. Mass Spectrom. 202 (2000) 217-229.

[9] K.J. Wu, R.W. Odom, Anal. Chem. 68 (1996) 573-882.

[10] K. Wittmaack, W. Szymczak, G. Hoheisel, W. Tuszynski, J. Am. Soc. Mass Spectrosc. 11 (2000) 553-563.

[11] S.L. Luxembourg, L.A. McDonnell, M.C. Duursma, X.H. Guo, R.M.A. Heeren, Anal. Chem. 75 (2003) 2333-2341.

[12] A.F.M. Altelaar, J. van Minnen, C.R. Jimenez, R.M.A. Heeren, S.R. Piersma, Anal. Chem. 77 (2005) 735-741.

[13] L.A. McDonnell, S.R. Piersma, A.F.M. Altelaar, T.H. Mize, S.L. Luxembourg, P.D.E.M. Verhaert, J. van Minnen, R.M.A. Heeren, J. Mass Spectrom. 40 (2005) 160-168.

[14] P. Sjovall, J. Lausmaa, H. Nygren, L. Carlsson, P. Malmberg, Anal. Chem. 75 (2003) 3429-3434.

[15] H. Nygren, P. Malmberg, C. Kriegeskotte, H.F. Arlinghaus, FEBS Lett. 566 (2004) 291-293.

[16] A. Delcorte, N. Medard, P. Bertrand, Anal. Chem. 74 (2002) 4955-4968.

[17] A. Delcorte, J. Bour, F. Aubriet, J.F. Muller, P. Bertrand, Anal. Chem. 75 (2003) 6875-6885.

[18] A. Delcorte, P. Bertrand, Appl. Surf. Sci. 231-2 (2004) 250-255.

[19] K. Keune, J.J. Boon, Surf. Interface Anal. 36 (2004) 1620-1628.

[20] L. Adriaensen, F. Vangaever, R. Gijbels, Anal. Chem. 76 (2004) $6777-$ 6785.

[21] J.A. Townes, A.K. White, E.N. Wiggins, K.D. Krantzman, B.J. Garrison, N. Winograd, J. Phys. Chem. A 103 (1999) 4587-4589.

[22] T.C. Nguyen, D.W. Ward, J.A. Townes, A.K. White, K.D. Krantzman, B.J. Garrison, J. Phys. Chem. B 104 (2000) 8221-8228.

[23] D. Weibel, S. Wong, N. Lockyer, P. Blenkinsopp, R. Hill, J.C. Vickerman, Anal. Chem. 75 (2003) 1754-1764.

[24] P. Sjovall, J. Lausmaa, B. Johansson, Anal. Chem. 76 (2004) 42714278.

[25] D. Touboul, F. Halgand, A. Brunelle, R. Kersting, E. Tallarek, B. Hagenhoff, O. Laprevote, Anal. Chem. 76 (2004) 1550-1559.

[26] P.J. Todd, J.M. McMahon, C.A. McCandlish, J. Am. Soc. Mass Spectrosc. 15 (2004) 1116-1122.

[27] A.F.M. Altelaar, I. Klinkert, K. Jalink, R.P.J. de Lange, R.A.H. Adan, R.M.A. Heeren, S.R. Piersma, Anal. Chem. 78 (2006) 734-742.

[28] L. Kuerschner, C.S. Ejsing, K. Ekroos, A. Shevchenko, K.I. Anderson, C. Thiele, Nat. Methods 2 (2005) 39-45.

[29] A.G. Sostarecz, D.M. Cannon, C.M. McQuaw, S.X. Sun, A.G. Ewing, N. Winograd, Langmuir 20 (2004) 4926-4932.

[30] R.P.J. De Lange, F.A. van Golen, J. van Minnen, Neuroscience 78 (1997) 289-299.

[31] B.W. Schueler, Microsc. Microanal. Microstruct. 3 (1992) 119-139.

[32] E.D. Schmidt, E.W. Roubos, Neuroscience 20 (1987) 247-257. 